\title{
Small Bowel Perforation Associated With Gastrointestinal Graft-Versus-Host Disease and Cytomegalovirus Enteritis in a Patient With Leukemia: A Case Report With Literature Review
}

\author{
Kwang-Seop Song ${ }^{1}$, Min Jung Kim², Han-Ki Lim ${ }^{1}$, Yoon Hwa Hong ${ }^{1}$, Sung Sil Park ${ }^{1}$, Chang Won Hong ${ }^{1}$, \\ Sung Chan Park ${ }^{1}$, Dae Kyung Sohn ${ }^{1}$, Kyung Su Han ${ }^{1}$, Jae Hwan Oh${ }^{1}$ \\ ${ }^{1}$ Center for Colorectal Cancer, Research Institute and Hospital, National Cancer Center, Goyang; ${ }^{2}$ Department of Surgery, Seoul National \\ University Hospital, Seoul National University College of Medicine, Seoul, Korea
}

Gastrointestinal graft-versus-host disease (GVHD) is a common complication after hematopoietic stem cell transplantation. Concomitant cytomegalovirus (CMV) enteritis worsens the prognosis of this condition. We report a case of small bowel perforation associated with gastrointestinal GVHD and CMV enteritis in a patient with leukemia who was successfully treated surgically. A 39-year-old man presented with intestinal perforation necessitating emergency surgical intervention. He was diagnosed with T-cell acute lymphoblastic leukemia and developed severe gastrointestinal GVHD and CMV enteritis after hematopoietic stem cell transplantation. His terminal ileum showed a perforation with diffuse wall thinning, and petechiae were observed over long segments of the distal ileum and the proximal colon. Small bowel segmental resection and a subtotal colectomy with a double-barreled ileocolostomy were performed. The patient recovered uneventfully after the operation. Based on reports described in the literature, surgery plays a minor role in the management of gastrointestinal GVHD; however, timely surgical intervention could be effective in selected patients.

Keywords: Graft vs host disease; Intestinal perforation; Surgical procedures

\section{INTRODUCTION}

Graft-versus-host disease (GVHD) is a common complication observed after hematopoietic stem cell transplantation [1]. The condition develops when the donor's immune system recognizes the recipient tissue as foreign. It usually involves the gastrointestinal tract, and patients present with diarrhea, abdominal pain,

Received: Jul 7, 2018 • Revised: Sep 30, 2018 • Accepted: Oct 1, 2018 Correspondence to: Min Jung Kim, M.D.

Department of Surgery, Seoul National University Hospital, Seoul National University College of Medicine, 101 Daehak-ro, Jongno-gu, Seoul 03080, Korea

Tel: +82-2-2072-2325, Fax: +82-2-766-3975, E-mail: minjungkim@snuh.org ORCID: https://orcid.org/0000-0002-3105-9633

(C) 2020 The Korean Society of Coloproctology

This is an open-access article distributed under the terms of the Creative Commons Attribution NonCommercial License (https://creativecommons.org/licenses/by-nc/4.0) which permits unrestricted noncommercial use, distribution, and reproduction in any medium, provided the original work is properly cited. nausea, and ileus [2]. Immunosuppressants are used for the treatment of gastrointestinal GVHD. Another complication occurring in patients undergoing transplantation is cytomegalovirus (CMV) enteritis, which mimics the clinical presentation of gastrointestinal GVHD [3]. Although it can be managed using antiviral agents, CMV enteritis occurring concomitantly with gastrointestinal GVHD worsens the prognosis in these patients [4].

Gastrointestinal GVHD is a medical condition with a poor prognosis. Thus, surgeons play a limited role in its management. Nevertheless, surgical intervention is inevitable in rare cases, such as in patients presenting with intestinal perforation. We report a case of bowel perforation following severe gastrointestinal GVHD and CMV enteritis with a review of the literature.

\section{CASE REPORT}

A 39-year-old man presented to a local Emergency Department with complaints of fever and chills. He reported dizziness and 
general weakness over the month prior to presentation. Blood tests revealed pancytopenia, and following a bone marrow study, he was diagnosed with T-cell acute lymphoblastic leukemia and referred to the National Cancer Center for hematopoietic stem cell transplantation. After induction and consolidation chemotherapy using daunorubicin, vincristine, prednisolone, L-asparaginase, etoposide, and methotrexate following total body irradiation over 5 months, he underwent unrelated hematopoietic stem cell transplantation with the administration of immunosuppressants and prophylactic antibiotics [5].

Approximately 2 months after transplantation, he was hospitalized due to newly developed nausea and vomiting. Esophagogastroduodenoscopic and colonoscopic examinations revealed edematous and hemorrhagic mucosal changes consistent with gastrointestinal GVHD (Fig. 1), and immediate treatment was initiated with an intravenously administered steroid. A punch biopsy examination performed at a later time revealed GVHD grade II. The patient's persistent symptoms necessitated the addition of the second-line immunosuppressants tacrolimus, cellcept, and cyclosporine A to his steroid regimen. Serial CMV antigenemia assays showed conversion from a negative to a positive status, and ganciclovir was added after a positive test result.

The patient suffered from persistent diarrhea, hematochezia, and abdominal pain, even after the administration of steroid and ganciclovir. On the 53th day after administration of steroid and the 28th day after ganciclovir, an abdominal computed tomography (CT) was performed because of the patient's severe continuing abdominal pain. CT showed definitive evidence of pneumoperitoneum, and a colorectal surgeon was consulted for an emergency laparotomy (Fig. 2). The patient was afebrile from the time

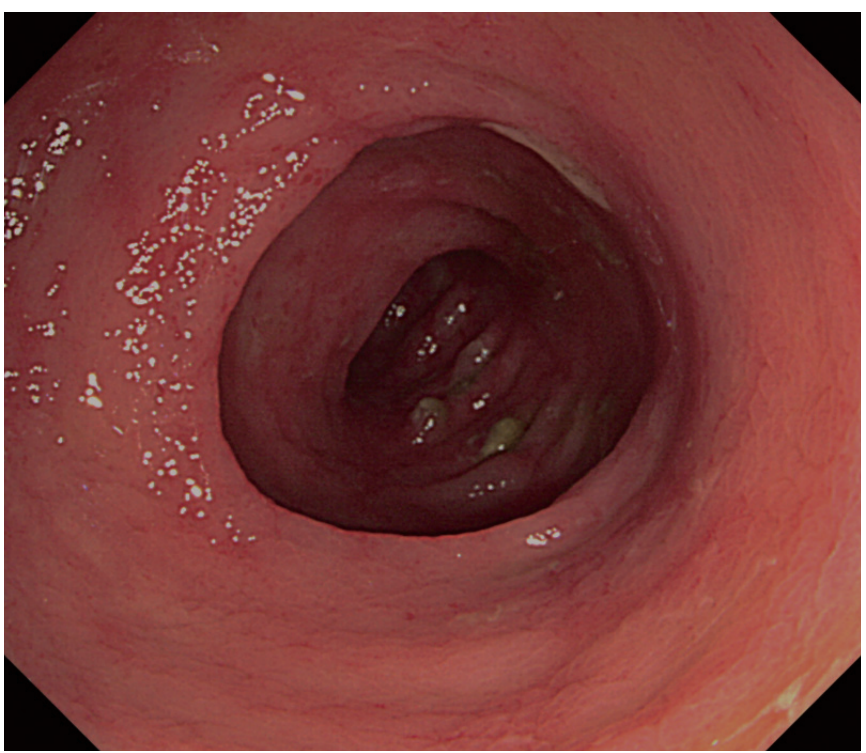

Fig. 1. Sigmoidoscopic examination revealed edematous and hemorrhagic mucosal changes consistent with graft-versus-host disease. of admission until the decision was made to perform the operation. However, preoperative laboratory tests indicated a systemic inflammatory reaction with a decreased absolute neutrophil count $(1,223 / \mu \mathrm{L})$ and an elevated C-reactive protein $(10.72 \mathrm{mg} / \mathrm{dL})$ level. Intraoperatively, a perforation was identified in the terminal ileum, and his abdominal cavity showed spillage of intestinal contents. The small bowel and colonic walls were observed to be very thin with multiple petechiae identified over long segments. Small bowel segmental resection $(70 \mathrm{~cm})$ and a subtotal colectomy with a double-barreled ileocolostomy were performed to remove all fragile and diseased segments (Fig. 3). After recovering from his poor general condition and underlying GVHD associated with CMV enteritis for 62 days, the patient was discharged with full recovery. GVHD associated with CMV enteritis was histopathologically confirmed (Figs. 4, 5). He underwent a double-barreled ileocolostomy takedown 6 months after the initial operation.

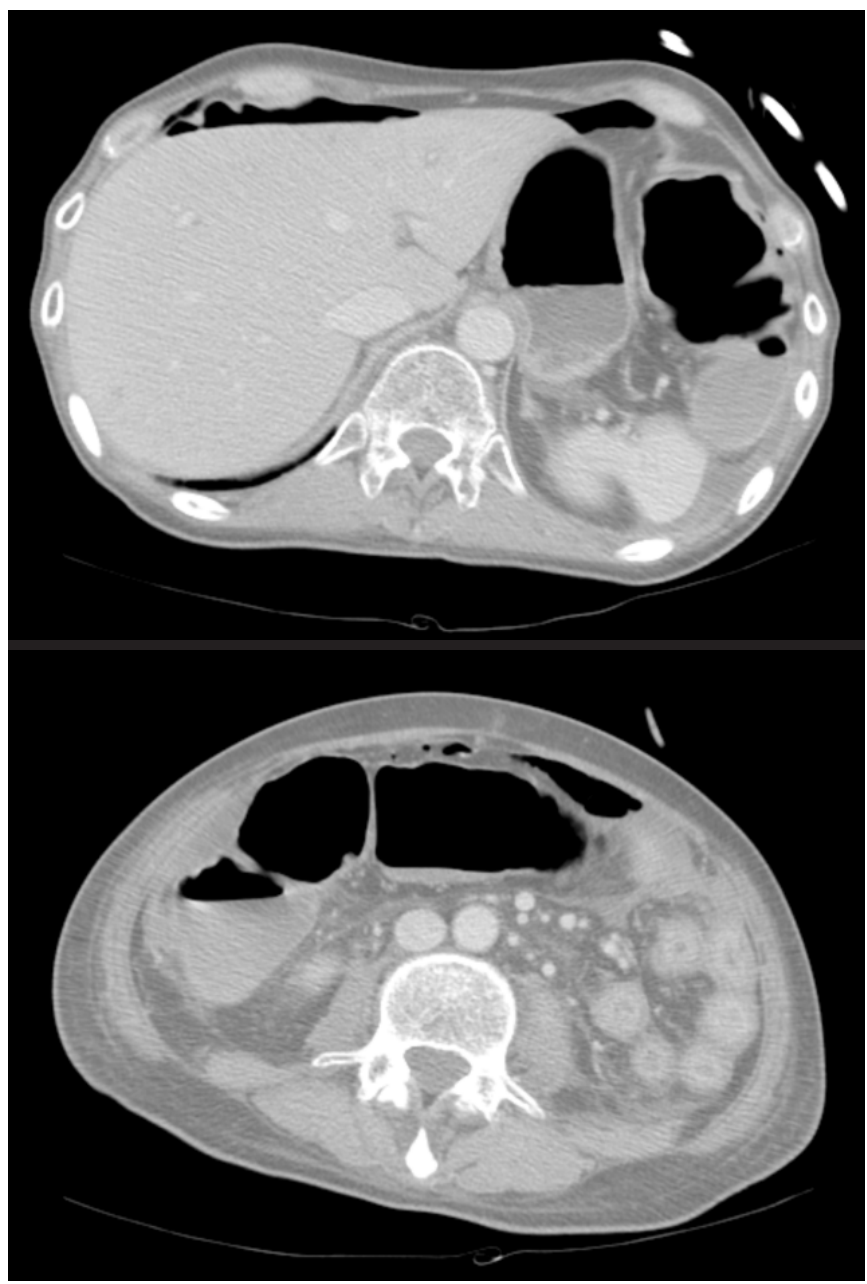

Fig. 2. Computed tomography scan showed pneumoperitoneum suggesting bowel perforation. The site of perforation could not be determined. 


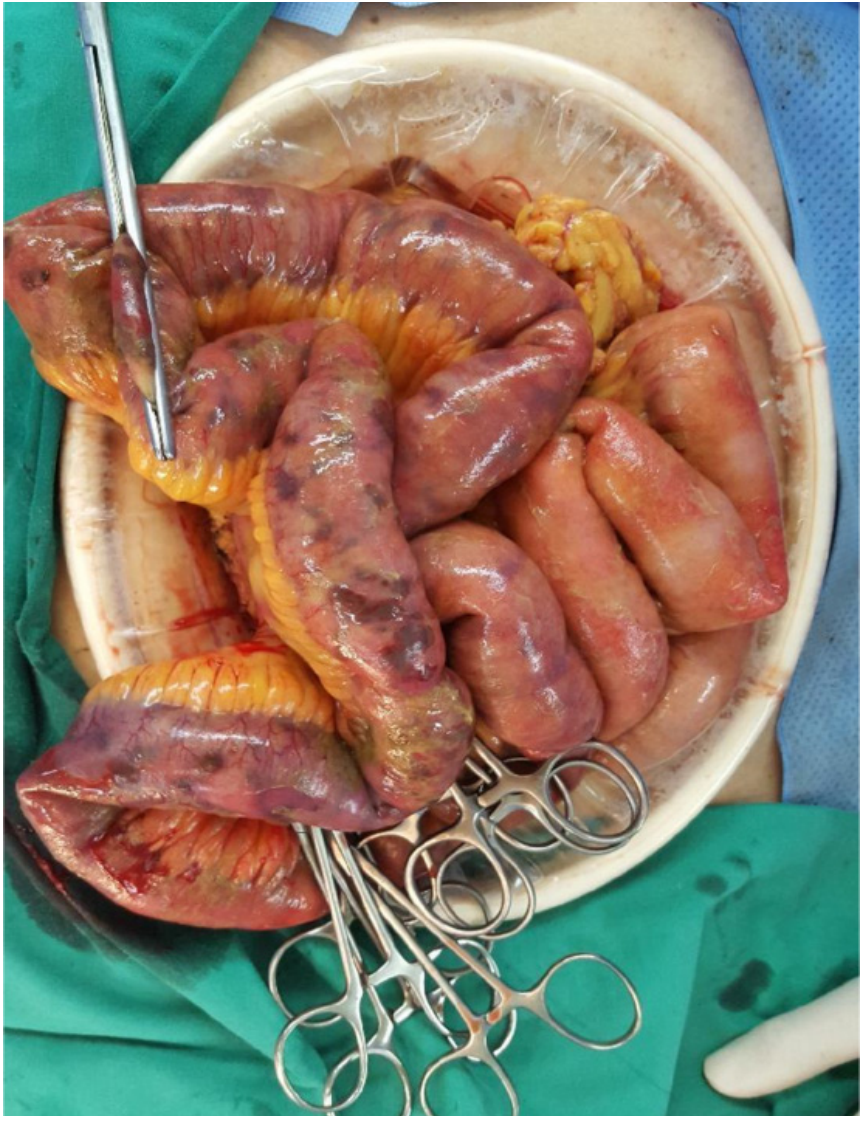

Fig. 3. Intraoperative findings showing a diseased small bowel segment.
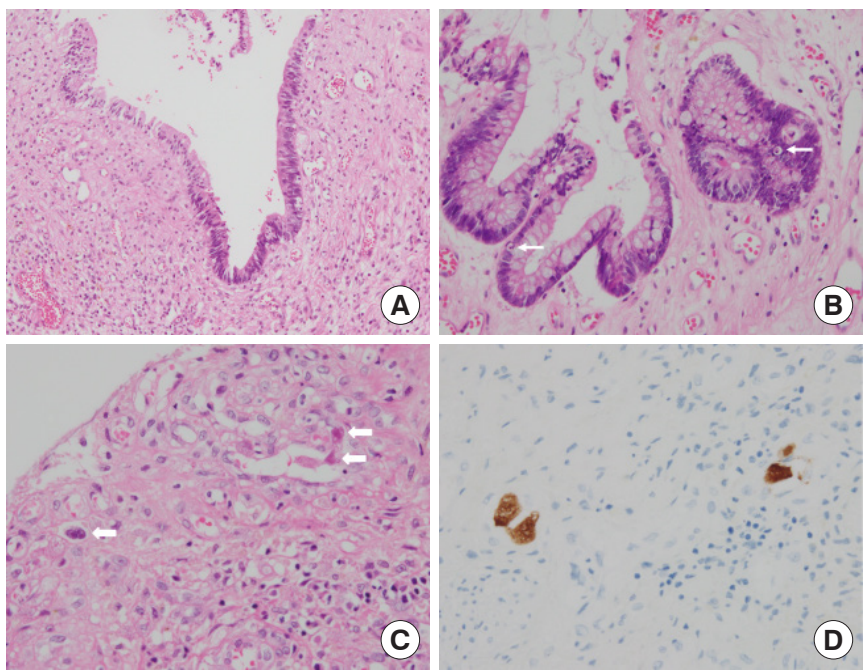

Fig. 5. Pathologic findings revealed flat mucosa $(A ; H \& E, \times 200)$ and apoptotic bodies within crypt epithelia (arrow) of graft-versus-host disease (B; $H \& E, \times 400)$, viral inclusions (bold arrow) (C: H\&E, $\times 400)$, and immunohistochemical staining of concomitant cytomegalovirus enteritis (D; immunohistochemistry for cytomegalovirus, $\times 400$ ).

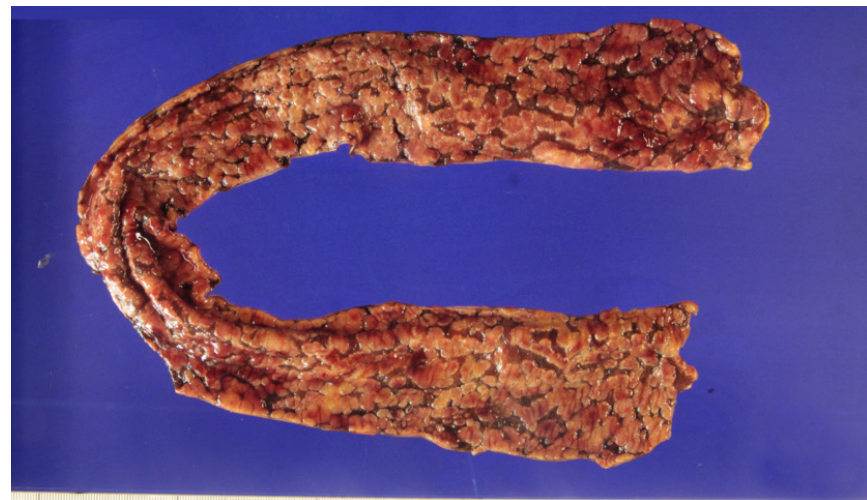

(A)

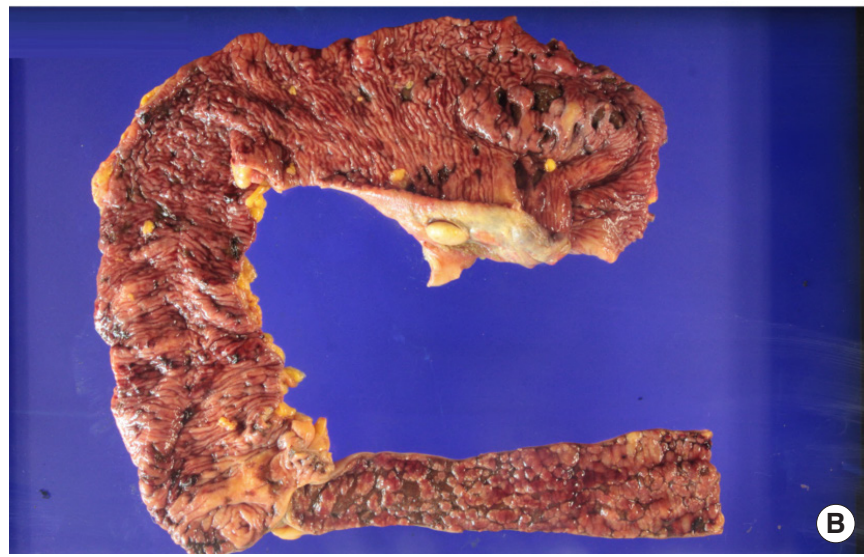

Fig. 4. Specimen from the small bowel segment (A) and the ascending colon with the terminal ileum (B) after the operation.

Informed consent was obtained from the patient, and the Institutional Review Board of National Cancer Center approved this case report (NCC2018-0105).

\section{DISCUSSION}

Gastrointestinal GVHD is a common complication after hematopoietic stem cell transplantation, developing in approximately $20 \%-30 \%$ of patients [6]. However, only a small percentage of patients present with a severe form of gastrointestinal GVHD [7]. Surgical management is warranted in patients with a clinical presentation of intractable intestinal hemorrhage, obstruction, pneumatosis intestinalis, and perforation [8-10]. However, a study reported that no surgically-correctable disease was observed in autopsies performed in 31 patients who died from severe gastrointestinal GVHD [7]. The authors concluded that surgical intervention is rarely required, and it was recommended that unnecessary operations be avoided.

Of note, bowel perforation remains a definitive indication for surgical intervention in patients presenting with severe gastrointestinal GVHD. Only a few case reports have described opera- 
tions for bowel perforation caused by severe gastrointestinal GVHD. A previous study reported operations for bowel perforation performed in 2 patients; one patient who had experienced an ileal perforation survived after ileocecal resection and side-to-side anastomosis, whereas the other patient died after total colectomy with an end ileostomy for cecal and left colonic microperforation [9]. Palaniappa et al. [6] reported multiple right colonic perforations in a patient who survived after a subtotal colectomy with an end ileostomy.

Severe gastrointestinal GVHD is associated with a poor prognosis $[11,12]$, which is worsened in those presenting with concomitant CMV gastroenteritis [4]. Additionally, most patients have an immune-compromised and poor general condition. Thus, surgical outcomes are likely to be disappointing. Unfortunately, owing to the limited number of cases studied, no conclusive guidelines have been established regarding the surgical principles to be followed for inevitable surgical indications. This lack of precedent cases and the diffuse nature of the disease lead to difficulties in decision making for surgeons regarding what type of surgery should be performed and the segment length of bowel that should be resected.

Resection of the thin, fragile, and diseased bowel segments, including the site of perforation, and a double-barreled ileocolostomy led to a recovery in our patient. Bowel perforation caused by severe gastrointestinal GVHD with CMV enteritis can be managed by a timely radical operation even in immunocompromised patients.

\section{CONFLICT OF INTEREST}

No potential conflict of interest relevant to this article was reported.

\section{REFERENCES}

1. Okubo H, Nagata N, Uemura N. Fulminant gastrointestinal graftversus-host disease concomitant with cytomegalovirus infection: case report and literature review. World J Gastroenterol 2013;19: 597-603.

2. Wang F, Cai B, Wang L, Gu Z, Luo L, Wei H, et al. Risk factors for acute graft-versus-host disease after allogeneic haematopoietic stem cell transplantation: a single-center experience. Ann Transplant 2017;22:58-65.

3. Cho BS, Yahng SA, Kim JH, Yoon JH, Shin SH, Lee SE, et al. Impact of cytomegalovirus gastrointestinal disease on the clinical outcomes in patients with gastrointestinal graft-versus-host disease in the era of preemptive therapy. Ann Hematol 2013;92:497504.

4. Bhutani D, Dyson G, Manasa R, Deol A, Ratanatharathorn V, Ayash L, et al. Incidence, risk factors, and outcome of cytomegalovirus viremia and gastroenteritis in patients with gastrointestinal graft-versus-host disease. Biol Blood Marrow Transplant 2015;21:159-64.

5. Yoon DH, Sohn BS, Lee WJ, Lim SN, Kim EK, Park I, et al. VPDL chemotherapy for T-cell lymphoblastic lymphoma (T-LBL) in adults: comparison with upfront autologous stem cell transplantation in a single center. Korean J Hematol 2008;43:138-44.

6. Palaniappa NC, Doyon L, Divino CM. Colonic perforation in graft versus host disease: a case report. Int Surg 2012;97:14-6.

7. Scoggins CR. Severe acute gastrointestinal graft-vs-host disease. Arch Surg 2008;143:1046.

8. Faraci M, Dallorso S, Morreale G, Dini G, Castagnola E, Miano M, et al. Surgery for acute graft-versus-host disease of the bowel: description of a pediatric case. J Pediatr Hematol Oncol 2004;26: 441-3.

9. Chirletti P, Caronna R, Arcese W, Iori AP, Calcaterra D, Cartoni C, et al. Gastrointestinal emergencies in patients with acute intestinal graft-versus-host disease. Leuk Lymphoma 1998;29:129-37.

10. Evans J, Percy J, Eckstein R, Ma D, Schnitzler M. Surgery for intestinal graft-versus-host disease: report of two cases. Dis Colon Rectum 1998;41:1573-6.

11. García-Cadenas I, Rivera I, Martino R, Esquirol A, Barba P, Novelli $S$, et al. Patterns of infection and infection-related mortality in patients with steroid-refractory acute graft versus host disease. Bone Marrow Transplant 2017;52:107-13.

12. Calmettes C, Vigouroux S, Labopin M, Tabrizi R, Turlure P, Lafarge $\mathrm{X}$, et al. Risk factors for steroid-refractory acute graft-versus-host disease after allogeneic stem cell transplantation from matched related or unrelated donors. Biol Blood Marrow Transplant 2015;21:860-5. 